\title{
A Rare Case of Pneumoperitoneum in Pregnancy: Perforation of Tubercular ileal Ulcer
}

\author{
Richmond Ronald Gomes
}

Associate Professor, Medicine, Ad-din Women's Medical College Hospital Dhaka, Bangladesh

*Corresponding Author: Richmond Ronald Gomes, Associate Professor, Medicine, Ad-din Women's Medical College Hospital Dhaka, Bangladesh

\section{Received date: June 19, 2021; Accepted date: August 21, 2021; Published date: October 30, 2021}

Citation: Richmond R. Gomes (2021). A Rare Case of Pneumoperitoneum in Pregnancy: Perforation of Tubercular ileal Ulcer. J. Obstetrics Gynecology and Reproductive Sciences; 5(8) DOI:10.31579/2578-8965/086

Copyright: (C) 2021, Richmond R. Gomes, This is an open access article distributed under the Creative Commons Attribution License, which permits unrestricted use, distribution, and reproduction in any medium, provided the original work is properly cited.

\begin{abstract}
:
The incidence of tuberculosis (TB) is rising worldwide, despite the efficacy of the BCG vaccination. Populations at greatest risk of contracting TB are migrant communities, as well as immunocompromised individuals. The diagnosis of intestinal tuberculosis can often present as a diagnostic conundrum, due to its nonspecific and varied presentation, often mimicking inflammatory bowel disease or malignancy. Free perforation is one of the most feared complications of the intestinal tuberculosis. The terminal ileum is the most common site of perforation, while the majority of (90\%) perforations are solitary. We present a 25 year old 17 weeks primi presented with peritonitis with solitary perforation of terminal ileum with miscarriage of fetus and subsequent surgical wedge resection of ileum and ileo-ileal anastomosis. Histology revealed presence of Langerhan's cell with caseating granulomatous inflammation. There was no radiological evidence of pulmonary tuberculosis. Patient was started on anti-tubercular therapy and responded well. This present case underscores the importance of biopsy specimens taken from the margins of patients with ileal perforation to avoid the misdiagnosis of such condition.
\end{abstract}

Key Words: intestinal tuberculosis; peritonitis; ileum; perforation; caseating granulomatous inflammation

\section{Introduction:}

TB remains a global epidemic, with an estimated 10.4 million new cases diagnosed worldwide in 2019. Of these, there was a slight male preponderance, $56 \%$ of all new cases, as compared to $34 \%$ for women. $\mathrm{TB}$ in children contributed to the remaining $10 \%$ of new cases. TB mortality rates are alarmingly high and in 2018 , approximately 1.8 million deaths were attributable to TB, amongst whom those coinfected with Human Immunodeficiency Virus (HIV) made up a quarter of total deaths. Perforation of a hollow viscus leading to peritonitis is a common surgical emergency. Although peptic ulcer perforations account for the majority of cases of peritonitis, small bowel perforations are also commonly encountered. Usually, these small bowel perforations are secondary to enteric fever or trauma, but at times, non- specific ileal perforations are also seen. Tuberculosis remains an uncommon cause of perforation of the small bowel, even in areas where tuberculosis is rampant. The incidence of intestinal perforation in patient with abdominal tuberculosis in close to that of 2.0\% recorded in one of the largest series from India [1-2]. Free intestinal perforation (4.9\%) is an uncommon complication of intestinal tuberculosis because of reactive thickening of the peritoneum and formation of adhesion with surrounding tissue [1-3]. It has a poor prognosis with mortality rate higher than 30\% [1-3]. Diagnostic and treatment modalities, even in pregnancy, are similar to the non-pregnant population. We report a case of 17 weeks pregnancy with a free tubercular perforation of the ileum that presented with peritonitis and was managed at our hospital.

\section{Case presentation:}

A 23 year old lady and 8 weeks into her first pregnancy when she commenced her antenatal care. Her Body Mass Index (BMI) at booking was only $17.23 \mathrm{~kg} / \mathrm{m}$, with a booking weight of $42 \mathrm{~kg}$. She had been experiencing intermittent, dull, right-sided lower abdominal pain that was associated with diarrhea along with low grade fever, for 3 months and in this span of time, her weight had decreased markedly by $8 \mathrm{~kg}$. Her primary healthcare provider at the district maternal and child health clinic subsequently treated her for acute gastroenteritis twice in the following weeks, but she experienced no improvement in her symptoms.

At 17 weeks of gestation, she was admitted under department of gynaecology and obstetrics of our hospital with the complaint of sudden onset of diffuse abdominal pain and vomiting for 1 day. She had been compliant to her oral hematinics therapy and denied any history of dysuria, constipation, haematuria, haematemesis, haematochezia., shortness of breath or night sweats. He denied any history of recent travel or contact with anyone infected with tuberculosis.

On physical examination, she appeared ill-looking, pale, afebrile and moderately dehydrated. Pulse was 120 beats/min, regular, blood pressure 90/60 $\mathrm{mm}$ of $\mathrm{Hg}$. On palpation, her fundal height corresponded to 16 
weeks of gestation. Abdomen was grossly distended with tenderness, rigidity and guarding were elicited diffusely all over abdomen. Bowel sound was sluggish. However, she did not exhibit any signs of preterm contraction and renal punch was negative. Transabdominal scan revealed a singleton fetus, with parameters corresponding to her gestation with no evidence of ruptured ectopic pregnancy. On the very next day she developed spontaneous preterm contractions and spontaneous abortion occurred.

She had hemoglobin of $9.4 \mathrm{~g} / \mathrm{dl}(\mathrm{MCV} 83.1, \mathrm{MCh} 28.8)$ and a white cell count of $5.87 \times 10^{9}$ with $\mathrm{N}: 48 \%$, L:45\%. As her pain worsened, repeat abdominal examination revealed obliteration of upper border of liver dullness and absent bowel sound. Serum lipase, random blood sugar, renal and liver function tests were non conclusive. X-ray abdomen in erect posture antero-posterior view showed free air under both the domes of the diaphragm (figure-1).She was kept nothing per oral. Parenteral fluid, analgesics and broad-spectrum antibiotic were commenced. She was also transfused with 1 unit of packed cells. An urgent ultrasound abdomen was arranged, which revealed a heterogeneous collection in the subhepatic region and multiple enlarged mesenteric lymph nodes. A diagnosis of likely perforated appendicitis was made and she underwent an emergency laparotomy.

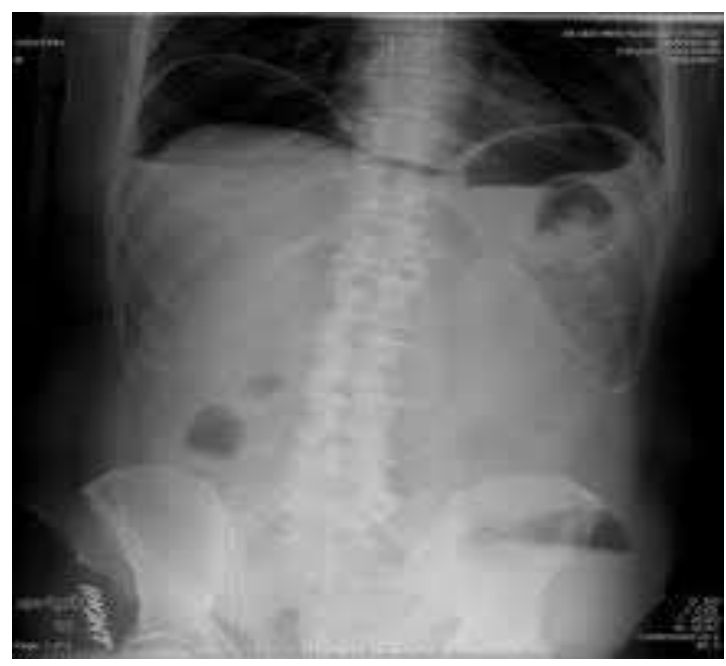

Laparotomy was performed by a midline incision and revealed purulent peritoneal fluid, mainly in the pelvis. The terminal ileum had a small perforation along the anti- mesenteric border, about one foot proximal to the ileocaecal junction. There were no other significant findings on laparotomy. There was evidence of multiple enlarged mesenteric lymph nodes. Ileal wedge resection with ileo-ileal anastomosis was done. Appendix was normal. The edges of the perforation were sent for histopathological examination.

The histopathological report of the perforation margins that were sent intra-operatively revealed caseating epithelioid cell granulomas with Langhans' giant cells and a dense mixed inflammatory cell infiltrate within the mucosa and submucosa (Figure-2). On receiving this report, the patient was started on anti-tubercular treatment. Her pulmonary TB work-up including chest X-ray, sputum for acid fast bacilli (AFB), and Mantoux test was negative. Screening tests for HIV were negative.

She was seen in outpatient clinic one-month post operatively and he was symptoms free.

She had put back most of her weight.

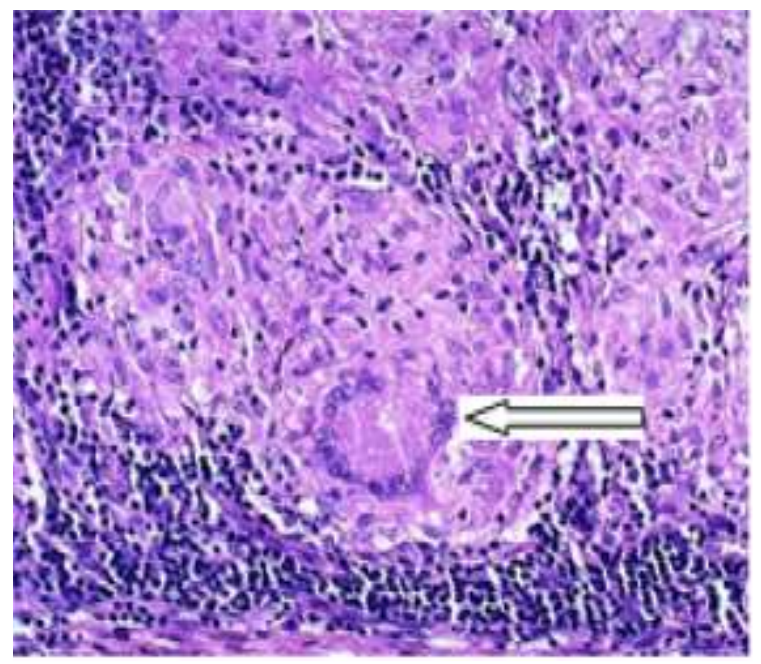

Figure 1: crescentic gas shadow under right dome of diaphragm and Figure 2: Margins of the perforation showing Langhans' giant cells and a dense mixed inflammatory cell infiltrate within the mucosa and submucosa.

\section{Discussion:}

Extrapulmonary tuberculosis (EPTB) is thought to be a result of reactivation of a prior dormant $\mathrm{TB}$ focus. Immunocompromised individuals have been found to be more susceptible to EPTB $^{16}$ Gastrointestinal TB is the sixth commonest site of extrapulmonary disease $[17,18]$ accounts for $11 \%$ of all EPTB. Its occurrence is attributed to four mechanisms: hematological, swallowing of infected sputum in patient with active pulmonary $\mathrm{TB}$, ingestion of milk or food contaminated with mycobacterium bovis, and contiguous transcoelomic spread from adjacent organs ${ }^{19}$.The ileocaecal region is affected in $75 \%$ of abdominal $\mathrm{TB}$ and this can present with perforation, abdominal mass, obstruction, and malabsorption $[6,16,20,21]$. Viscus perforation due to TB is very rare and occurs more commonly in immunosuppressed patients [22]. Pregnancy is a physiological state of relative immunosuppression. Hence, the risk of viscus perforation after contracting abdominal TB in pregnancy may be increased, as was the case in our patient.

Abdominal tuberculosis is common in Bangladesh and India, more in young [1]. Intestinal tuberculosis can occur both primary and secondary to a tuberculous focus at different sites [8]. Primary intestinal tuberculosis (without pulmonary involvement) is one of the commonest forms of extrapulmonary tuberculosis. The infection is usually caused by ingestion of unpasteurized or contaminated milk contaminated by Mycobacterium bovis, that leads to a primary infection of the intestine in the absence of pulmonary disease[4].The tubercle bacillus is protected against digestion in the stomach by its fatty capsule .Intestinal tuberculosis commonly affects the ileocaecal region because of the following reasons: 1) the terminal ileum is an area of physiological stasis; 2) it has abundant lymphoid tissue; and 3) it has a high absorptive capacity. Thus, after the initial infection occurs in the Peyer's patches, mucosal edema and sloughing occur, leading to the formation of typical tubercular ulcers that lie transversely to the long axis of the ileum. The disease may spread further by dissemination through the lymphatics and by caseation, may heal by fibrosis, or may even remain confined to the area if the host's defence mechanisms are adequate [5].

The complications of intestinal tuberculosis are bowel obstruction $(31.7 \%)$, intestinal perforation (4.9\%), enterocutaneous fistula $(2.4 \%)$ and small bowel volvolus due to mesenteric lymphadenitis (2.4\%)[10].There are three main pathological forms of tubercular enteritis: 1) ulcerative, 2) hypertrophic, and 3) ulcerohypertrophic ${ }^{5}$. The ulcerative form of the 
disease is more common than the others, but these ulcers rarely perforate. Fibrosis and the formation of adhesions to adjacent intra-abdominal organs account for the low incidence of perforation seen in tuberculosis $[2,5]$. However, if perforation occurs, the patient presents with the signs and symptoms of peritonitis. Although it is well documented that the incidence of perforation in patients with intestinal tuberculosis varies from $1 \%$ to $11 \%$, the majority of these perforations $(70 \%-80 \%)$ are not truly perforations of such tubercular ulcers, but are 'blow outs' of the small bowel secondary to distension due to distal obstruction (strictures or adhesions). As such, true or 'free' perforations are rare, and only a few cases have been reported to the present in the world literature, with an overall mortality rate of nearly $70 \%$. Recently, vasculitis of the mesenteric vasculature due to tuberculosis has been implicated as a contributory factor, but the exact mechanism by which some patients develop perforation and others is not established [5,6,7] . A single perforation is present in $90.0 \%$ of cases [2].Multiple perforations occur in $40.0 \%$ of patients and are associated with poor prognosis[9].

Caseating necrosis surrounded by chronic inflammatory tissue is the hallmark of tuberculosis. The characteristic histopathology is seen more often in affected lymph node than in the bowel $[8,11]$. The symptoms anaemia, lymphocytosis, and elevated ESR, tuberculin test positive in $42.0 \%$ cases, are common in patient with tuberculosis [1, 8]. Radiographic evidence of free gas is found in only $25.0 \%$ cases [2]. Thirty to $50.0 \%$ of patients with abdominal tuberculosis have a normal chest film [1]. The definite diagnosis of tuberculosis can be made by demonstration of Mycobacterium tuberculosis in lesion and growth in culture or presence of typical histopathological findings [8]. Makanguola has shown that computed tomography can provide a diagnosis of intestinal tuberculosis in $81 \%$ of the cases [12].

The treatment of tubercular peritonitis is similar to that for peritonitis due to other causes like resuscitation, nasogastric aspiration, intravenous fluids, antibiotics, and surgery once the patient is stabilized. Tubercular perforation is rarely diagnosed pre-operatively as the signs and symptoms are similar to those of peritonitis and there are no pathognomic features either on investigation or on clinical examination. Even in patients who are known to be sufferers of the disease, the diagnosis of perforated tubercular ulcers cannot be made with certainty. Primary closure of the perforation can be considered safe if the patient has presented early and the bowel is healthy, otherwise, exteriorization of the affected bowel as a loop ileostomy is a safer option. If there is a long segment of bowel that is diseased, or there are multiple perforations, resection with either primary anastomosis or exteriorization may be considered. Removing the appendix is a safe procedure even if microscopic evidence of tuberculosis is present [2]. Simple repair of the perforation is not recommended because of the high incidence of leak and fistula formation [14]. High mortality and morbidity reported more than $(29.3 \%)$ but lower mortality is seen inpatients operated within 36 hours of perforation [15]. Once biopsy confirms the diagnosis of tuberculosis of the bowel, antitubercular therapy is mandatory. Rationale most of the guidelines on the treatment of tuberculosis suggest that 6 months treatment is sufficient for abdominal tuberculosis [13, 14]. In a randomized controlled trial, Balasubra-maniam et al [15]. Reported no difference in success rate of 6 months $(99.0 \%)$ versus 12 months $(94.0 \%)$ anti tuberculous drugs (conventional strategy) in the treatment of abdominal tuberculosis. They also need to be followed up in Chest as well as GI clinic to monitor any future recurrence.

As this condition is uncommon, it is important to send the margins of any perforation routinely for histopathological analysis, especially in areas where tuberculosis is endemic. We realized that a potentially treatable disease like tuberculosis can be missed by omitting a biopsy, since we consider such perforation is secondary to enteric fever or peptic ulcer even in our institution. If tuberculosis is suspected intra-operatively, any other suspicious tissues (e.g. lymph nodes, fluid) should also be analyzed, as the combination of histology and culture helps to establish the diagnosis in nearly $80 \%$ of the cases [7]. Another important point to keep in mind is the association of tuberculosis with HIV infection, and such patients must always be screened for HIV if the diagnosis of tuberculosis is made.

\section{Management in Pregnancy:}

A high index of suspicion is required in at-risk populations, such as ethnic minorities, migrant communities, and immunocompromised individuals. Tuberculin skin testing is a valuable screening test in pregnancy and should be carried out if latent TB is suspected. Imaging modalities such as chest X-ray with shielding, CT scan, or ultrasound can be performed as necessary [22].

Pregnancy does not affect the course of TB. However, any delay in diagnosis and treatment could result in catastrophic repercussions to mother and baby, such as the resultant viscus perforations and severe preterm delivery or spontaneous abortion as seen in our case described or even mortality. Intrauterine growth restriction and vertical transmission have also been described [22]. Coinfection with HIV increases maternal mortality rates [22].

First-line anti tuberculous drugs such as isoniazid, rifampicin, and ethambutol can be used safely in pregnancy and while breastfeeding.

EPTB is an important diagnosis to be considered in high risk women with a nonspecific presentation of abdominal pain diarrhoea and chronic constitutional symptoms, as in our case. Diagnostic delay can lead to unnecessary maternal and fetal morbidity and mortality [23].

In our case described, a pregnant lady presenting with chronic constitutional symptoms with abdominal pain and diarrhoea should have been thoroughly investigated for possible causes, at primary contact, with referral to the obstetrics and medical teams for specialist care.

Normochromic normocytic anaemia, which does not respond to oral iron therapy, warrants further investigations to look for chronic blood loss, chronic disease, and malignancy. With an earlier referral, a biopsy might have sufficed, with consequent medical anti-TB therapy commenced. Viscus perforation may have been avoided, with resultant less morbidity for mother.

\section{Conclusion:}

Tuberculosis remains an important cause of mortality and severe morbidity in the world, despite the availability of sound Bacillus Calmette-Guerin (BCG) vaccination programmes in most countries. It is often difficult to differentiate EPTB from Crohn's disease or malignancy. Diagnosis of acute ileal tuberculous perforation is difficult because of the rarity of such case and consequent tendency to concentrate on other causes of viscus perforation as perforated duodenal ulcer. In endemic areas, clinicians should stay vigilant of this condition as a possible differential in patients presenting with acute or chronic abdominal pain. Prompt diagnosis with urgent surgical intervention and continuing medical treatment can decrease the morbidity and mortality.

\section{Funding: None.}

Ethical approval: Not applicable.

Conflicts of interest: The authors have no competing interests relevant to the present study.

\section{References:}

1. Sarkar DN, Amin R, Mohammed H, Azhar MA, (2011) Faiz MA. Abdominal tuberculosis- A Review. Bangladesh J Med; 22: $51-59$ 
2. Desgupta A, Singh N, Bhatia A. (2009) Abdominal Tuberculosis: A Histopathological Study with Special Reference to Intestinal Perforation and Mesenteric vasculopathy. J Lab Physicians;1(2):56-61

3. Saleh MAS, Hayan B. (2002) Acute ileal tuberculosis perforation: A case report. Saudi J Gastroenterol;8(2):64-66

4. Sefr R, Rotterova P, Konecny J. (2001) Perforation peritonitis in primary intestinal tuberculosis. Dig Surg. ;18:475-479.

5. Gupta RL. (2001) Abdominal Tuberculosis. In: Chattopadhya TK, editor. GI Surgery Annual. Vol. 2. New Delhi: Saku Printing House; pp. 51-60.

6. Bhansali SK. (1997) Abdominal tuberculosis. Experiences with 300 cases. Am J Gastroenterol.;67:324-337.

7. Shah S, Thomas V, Mathan M, Chacko A, Chandy G, Ramakrishna BS, (1992) et al. Colonoscopic study of 50 patients with colonic tuberculosis. Gut. ;33:347-351.

8. Gupta M, Jayent M, Kaushik R. (2013) Free tubercular perforation of the ileum. World J Emerg Med;4(3):235-236

9. Makharia GK, Ghoshal UC, Ramakrishna BS, Agnihotri A, Ahuja V, Chowdhury SD et al. (2015) Intermittent Directly Observed Therapy for Abdominal tuberculosis : A Multicenter Randomized Controlled Trial Comparing 6 months versus 9 months of therapy. Clin Infect Dis;61(5):750-757

10. Alper Akino Glu, Ilter Bilgin. (1988) Tuberculous Enteritis and Peritonitis. Can J Surg, 31: 558.

11. Coccolini F, Ansaloni L, Catena F, Lazzareschi D, Puviani L, (2011) Pinna AD. Tubercular bowel perforation: What to do? Turkish J Trauma Emerg Surg;17(1):66-74
12. Makanjuola D, (1998) Is it Crohn's disease or intestinal T.B? CT analysis. Eur-J-Radiol,28(1): 55-61.

13. Aston NO, (1985) Decosta AM. Tuberculous perforation of the small bowel. Postgraduate Med $J$;61:251-252

14. Pennathur A. (1994) Tuberculosis of small intestine. Surg Tropical Dis; 24-92

15. Talwar S, Talwar R, Prasad P (1999) Tuberculous perforations of the small intestine. Int J Clin Pract 53: 514-518.

16. Kapoor V. K., Pravin R., Pravir G. (2016) Abdominal tuberculosis. Journal of The Association of Physicians of India. February;64

17. Ved Bhushan S. T., Mulla M. A., Kumar V. (2015) Ruptured appendix in tuberculous abdomen. Biological System. ;4(1):1-6.

18. Paustian F. (1964) Tuberculosis of the intestine. In: Bockus H. L., editor. Gastroenterology. 2nd. Vol. 311. Philadelphia, Pa, USA: W.B. Saunders Co.

19. Nisar A., Muhammad H., Muhammad I. (2011) Tuberculosis as a cause of small bowel obstruction in adults. Gomal Journal of Medical Sciences.;9, article 233(2)

20. Bhansali S. K. (1977) Abdominal tuberculosis. Experiences with 300 cases. American Journal of Gastroenterology. ;67:324-337.

21. Prakash A. (1978) Ulcero-constrictive tuberculosis of the bowel. International Surgery. ;63:23-29.

22. Mahendru A., (2011) et al. Review Diagnosis and Management of tuberculosis in pregnancy. The Obstetrician \& Gynaecologist ;12(3)

23. Kangeyan N., Webster S. N. E., Sanyal A., (2012) et al. Tuberculosis in pregnancy - diagnostic dilemma. Open Journal of Obstetrics and Gynecology ;2:174-175. 